\title{
Levantamento de solos utilizando geoestatística em uma área de experimentação agrícola em Petrolina-PE
}

\author{
Kátia Araújo da Silva, Marcos Sales Rodrigues*, Jailson Cavalcante Cunha, David Castro Alves, \\ Helder Ribeiro Freitas, Augusto Miguel Nascimento Lima
}

Universidade Federal do Vale do São Francisco, Petrolina, PE, Brazil

*Autor correspondente, e-mail: marcos.rodrigues@univasf.edu.br

\section{Resumo}

Dada a importância de informações precisas sobre os solos para a produção agrícola, técnicas quantitativas para estimar classes e/ou propriedades dos solos têm sido desenvolvidas para aumentar a qualidade destes mapas. Objetivou-se neste trabalho mapear solos em nível ultradetalhado em uma área de experimentação agrícola com o uso de ferramentas geoestatísticas a fim de auxiliar na alocação e manejo de experimentos. O local de estudo se localiza em uma área de experimentação agrícola, no Campus de Ciências Agrárias da Universidade Federal do Vale do São Francisco, município de Petrolina-PE. O mapeamento da área constituiu-se de um perfil completo e uma malha amostral com 51 pontos georreferenciados. Na malha foram realizadas amostragens deformadas nas camadas de 0-0,2 $\mathrm{m}$ e 0,4-0,6 $\mathrm{m}$ de profundidade que corresponderam aos horizontes $\mathrm{A}$ e $\mathrm{B}$, respectivamente. Foram realizadas as análises físicas (granulométrica) e químicas do solo. Descreveu-se a dependência espacial dos atributos do solo usando-se semivariogramas e os mapas foram interpolados pela krigagem ordinária. Os resultados mostraram que os solos predominantes na área foram Argissolo Amarelo eutrocoeso típico, Argissolo Amarelo distrocoeso típico e Argissolo Amarelo distrocoeso arênico. A geoestatística demonstrou ser uma ferramenta importante no auxílio do mapeamento pedológico no nível ultradetalhado da área de experimentação agrícola.

Palavras-chave: análise espacial, krigagem, pedometria, semiárido

\section{Soil survey using geostatistics in an agricultural experimental area in Petrolina, Pernambuco state, Brazil}

\begin{abstract}
Due to the importance of accurate information on soils for agricultural production, quantitative techniques to estimate classes and/or soil properties have been developed to increase these maps quality. This manuscript was developed to map soils of an agronomic experimental area using geostatistical tools to assist in the allocation and management of experiments. The study area is located in an agricultural experimental area of Federal University of São Francisco Valley, Petrolina County, Pernambuco state, Brazil. Mapping the agricultural area consisted of a soil profile and a sampling grid with 51 georeferenced points. At these points the soil samples were collected at 0-0.2 $\mathrm{m}$ and $0.4-0.6 \mathrm{~m}$ soil depths that corresponded to A and B soil horizons, respectively. Soil samples were evaluated for physical (soil texture) and chemical analysis. The spatial dependence of soil attributes was described using semivariograms and maps were interpolated by ordinary kriging. The results showed that the predominant soils in the area were Ultisol with a cohesive eutrophic horizon, Ultisol with a cohesive dystrophic horizon, and Ultisol with a sandy cohesive dystrophic horizon. The geostatistical proved to be an important tool supporting the ultra-detailed pedological mapping of the agricultural research area.
\end{abstract}

Keywords: spatial analysis, kriging, pedometry, semi arid 
O levantamento de solos é o termo mais frequentemente usado para descrever a distribuição espacial de solos em áreas agrícolas. As metodologias utilizadas no levantamento de solos visam à produção de mapas de classes e atributos com maior nível de detalhamento para que sejam aplicados para fins de manejo e conservação do solo, planejamento de uso racional das terras, previsão de cenários futuros, extrapolação de resultados de pesquisa e fonte de dados para modelagem espaço-temporal (Dalmolin \& Ten Caten, 2015; Shi et al., 2006).

Todavia, o levantamento de solos tradicional não apresenta informações precisas, pois se baseia num conhecimento tácito e intuitivo, também não apresenta confiabilidade das interpretações excessivamente qualitativas e existindo ainda a dificuldade de extrapolar as informações obtidas para outras áreas (Silva et al., 2013). Assim sendo, tem-se a necessidade do uso de novas tecnologias e modelos quantitativos, surgindo então um ramo da Ciência do Solo denominado Pedometria (Dalmolin \& Ten Caten, 2015), a qual é definida por Alex McBratney da Universidade de Sydney como a ciência baseada em métodos matemáticos, estatísticos quantitativos e numéricos que objetiva analisar a variação espaço-temporal, propriedades e comportamento dos solos e gerar informações espacialmente localizadas de atributos e classes de solo (Ten Caten et al., 2012).

A geoestatística é uma das técnicas quantitativas aplicada para integrar 0 mapeamento convencional do solo, a qual busca entender a aparente aleatoriedade dos dados que, por apresentarem uma possível estruturação espacial, estabelece uma função de correlação espacial. A técnica de interpolação krigagem tem se destacado na produção de mapas por considerar a dependência espacial das amostras através do semivariograma (Oliver \& Webster, 2014). Portanto, objetivou-se nesse trabalho mapear solos de uma área experimental agrícola com o uso de ferramentas geoestatísticas a fim de auxiliar na alocação e manejo de experimentos.

O estudo foi conduzido em uma área de experimentação agrícola da Universidade Federal do Vale do São Francisco, Campus de Ciências Agrárias, situada no município de Petrolina-PE, a qual está localizada nas coordenadas geográficas $9^{\circ} 19^{\prime} 10,47^{\prime \prime}$ de latitude Sul e 40³3'48,91" de longitude Oeste, altitude média de $375 \mathrm{~m}$, relevo plano, inserida na região do Vale do Submédio São Francisco, compreendendo 24,7 hectares. Segundo a classificação de Köppen, o clima local é do tipo Bsh', semiárido, precipitação inferior a $500 \mathrm{~mm}$ concentrada em três a quatro meses do ano, com médias anuais de temperaturas variando entre 18,7 e $33,6^{\circ} \mathrm{C}$ (mínima e máxima, respectivamente).

Para que um levantamento pedológico seja classificado como ultradetalhado, é necessário que o número de perfis abertos esteja entre 1 perfil a cada 10-30 ha e o número de amostras extras esteja entre 1 amostra a cada 0,25-1 ha (IBGE, 2015). No mapeamento da área agrícola da Univasf foi realizada a abertura de um perfil ( $1 / 24,7$ ha) e 51 amostras extras ( 1 amostra a cada 0,48 ha) espaçados em aproximadamente $32 \mathrm{~m}$. Utilizaram-se, como método de prospecção, malhas rígidas definidas através do uso de um sistema de informações geográficas (SIG) e coletaram-se as amostras por meio de um receptor GPS ('Global Positioning System') de navegação Garmin Etrex 20 com erro médio de $3 \mathrm{~m}$.

Foi descrito um perfil representativo da classe de solo (perfil modal) para descrição geral e morfológica do solo, a qual seguiu a metodologia descrita no Manual de descrição e coleta de solo a campo (Santos et al., 2013a).

Nos 51 pontos de amostragem foram coletadas amostras deformadas nas camadas de 0-0,2 $\mathrm{m}$ e 0,4-0,6 $\mathrm{m}$ de profundidade, seguindo a malha amostral previamente estabelecida, utilizando um trado tipo holandês. Esta profundidade foi baseada na avaliação do perfil, onde corresponde respectivamente aos horizontes A e B do solo.

As análises nestas amostras foram: a textura pelo método da pipeta, o pH do solo em água (relação 1:2), condutividade elétrica $(C E)$, a acidez potencial $(H+A l)$, acidez trocável $\left(\mathrm{A}^{\beta^{++}}\right)$e os cátions trocáveis $\left(\mathrm{Ca}^{2+}, \mathrm{Mg}^{2+}, \mathrm{K}^{+} \mathrm{e} \mathrm{Na}^{+}\right.$), conforme metodologia descritas por Donagema (2011). 
Os dados foram submetidos à análise estatística descritiva (valores médios, máximos, mínimos e coeficiente de variação). Modelos de semivariogramas foram ajustados aos dados para a descrição da dependência espacial entre as amostras (Oliver \& Webster, 2014). As informações geradas pelos semivariogramas foram usadas na interpolação de dados para a produção de mapas pelo interpolador krigagem ordinária. Para as variáveis que apresentaram tendência, foi realizada a remoção da tendência pela subtração de uma superfície ajustada por mínimos quadrados, conforme descrito por Vieira et al. (2010). A escolha de quais mapas utilizar na obtenção do mapa pedológico foi dependente da análise do perfil para a definição do terceiro e quarto nível categórico. Todos os mapas foram editados no software ArcGis Trial License.

A análise morfométrica do perfil (Tabela 1), assim como a análise laboratorial das amostras retiradas nos horizontes determinados no campo (Tabela 2), permitiu verificar que o solo é um Argissolo Amarelo distrocoeso típico A moderado, relevo plano, textura arenosa. No primeiro nível categórico o solo foi classificado como Argissolo já que a fração argila aumentou em profundidade no perfil estudado, resultando em um gradiente textural, razão B/A de 2,04, caracterizando a presença do horizonte $B$ textural (Bt) (Santos et al., 2013b).

Tabela 1. Atributos morfológicos e físicos do perfil modal de um Argissolo Amarelo distrocoeso típico de uma área de experimentação agrícola em Petrolina-PE.

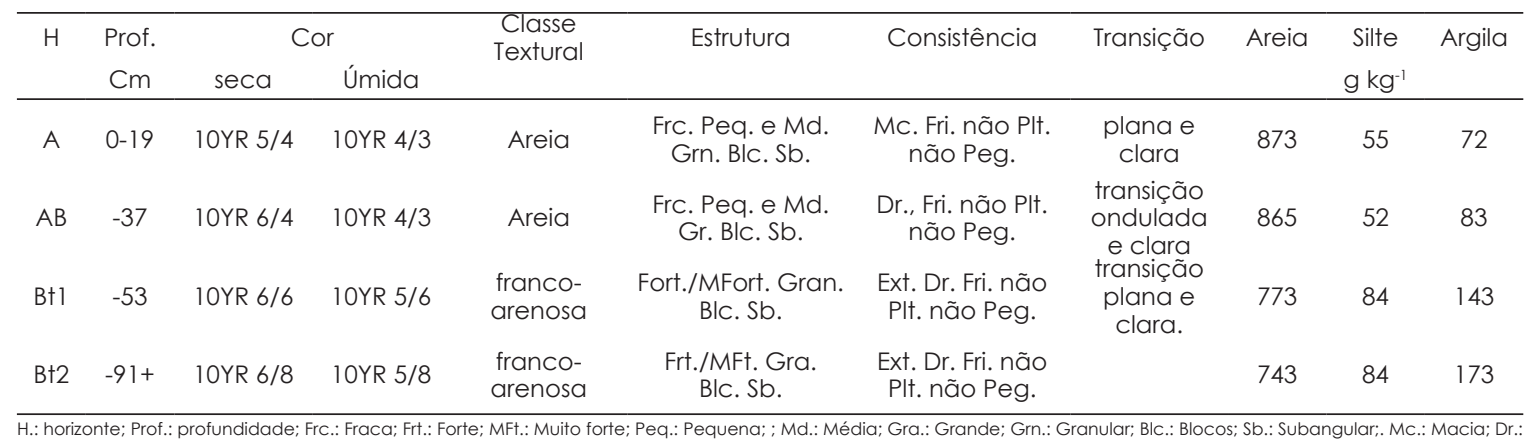

Dura; Ext.: Extremamente; Fri.: Friável; Plt.: Plástica; Peg.: Pegajosa.

Tabela 2. Atributos químicos do perfil modal de um Argissolo Amarelo distrocoeso típico de uma área de experimentação agrícola em Petrolina-PE.

\begin{tabular}{|c|c|c|c|c|c|c|c|c|c|c|c|c|c|}
\hline $\mathrm{H}$ & Prof. & \multicolumn{2}{|c|}{$\mathrm{pH}$} & $\mathrm{Ca}^{2+}$ & $\mathrm{Mg}^{2+}$ & $\mathrm{K}^{+}$ & $\mathrm{Na}^{+}$ & SB & $\mathrm{Al}^{3+}$ & $T$ & $\mathrm{~V}$ & $m$ & Corg. \\
\hline & & $\mathrm{H}_{2} \mathrm{O}$ & $\mathrm{KCl}$ & \multicolumn{7}{|c|}{$\mathrm{cmol}_{\mathrm{c}} \mathrm{kg}^{-1}$} & \multicolumn{2}{|c|}{$\%$} & $\mathrm{~g} \mathrm{~kg}^{-1}$ \\
\hline$A$ & $0-19$ & 5,5 & 4,7 & 1,76 & 0,23 & 0,15 & 0 & 2,14 & 0,21 & 3,67 & 58,31 & 8,94 & 9 \\
\hline$A B$ & -37 & 5,5 & 4,0 & 0,72 & 0,11 & 0,10 & 0 & 0,93 & 0,25 & 3,26 & 28,53 & 22,12 & 4 \\
\hline $\mathrm{B}+1$ & -53 & 5,2 & 3,8 & 0,62 & 0,06 & 0,05 & 0 & 0,73 & 0,62 & 3,06 & 23,86 & 44,29 & 3 \\
\hline $\mathrm{B}+2$ & $-91+$ & 4,4 & 3,6 & 0,51 & 0,02 & 0,03 & 0 & 0,56 & 0,91 & 2,41 & 23,24 & 61,07 & 2 \\
\hline
\end{tabular}

Conforme Santos et al. (2013b), a cor permite classificar os Argissolos ao $2^{\circ}$ nível categórico (subordem), diante disto o solo foi classificado como Amarelo, o qual representa solos com matiz 7,5YR ou mais amarelo na maior parte dos primeiros $100 \mathrm{~cm}$ de horizonte B. O horizonte $\mathrm{B} \dagger$ foi considerado coeso já que atendeu aos critérios de serem muito resistentes à penetração da faca e extremamente duros quando seco, passando a friáveis quando úmido (Santos et al., 2013b).

Para a determinação do terceiro nível categórico $\bigcirc$ atributo diagnóstico utilizado foi a saturação por bases $(V)$ que poderia diferenciar o solo em Eutrocoeso e Distrocoeso. No quarto nível categórico a textura foi o atributo diagnóstico utilizado para diferenciar solos arênicos de típicos. Na tabela 3 são apresentados as estatísticas descritivas e os parâmetros dos semivariogramas das variáveis analisadas, contudo para a confecção do mapa das classes de solos utilizaram-se os mapas interpolados pela krigagem da $V$ e textura do solo, visto que estas variáveis determinaram o $3^{\circ}$ e $4^{\circ}$ níveis categóricos.

Entre as variáveis utilizadas para a produção do mapa de classes de solo ( $\mathrm{V}$ e textura) todas apresentaram dependência 
espacial (Tabela 3). O mapa de saturação por bases na camada 0,4-0,6 $\mathrm{m}$ de profundidade possibilitou a identificação do $3^{\circ}$ nível categórico (grandes grupos), separando-os em Eutrocoeso $(V>50 \%$ ) e Distrocoeso ( $V<50 \%)$. Vale ressaltar que a escolha de considerar os dados apenas da $2^{a}$ camada (horizonte B), embora seja necessário conhecer as características do solo dos primeiros $100 \mathrm{~cm}$, ocorreu devido a grande variabilidade de uso do solo na área, com diferentes culturas e, consequentemente, diferentes manejos tais como correções e adubações do solo, ações antrópicas que poderiam mascarar o valor de saturação por base natural do solo.

Tabela 3. Estatística descritiva e parâmetros do modelo semivariograma de atributos dos horizontes $A$ e $B$ das amostras extras de um Argissolo em uma área de experimentação em Petrolina-PE.

\begin{tabular}{|c|c|c|c|c|c|c|c|c|c|}
\hline \multirow{2}{*}{ Variável } & Méd. & Mín. & Máx. & $\begin{array}{l}\text { CV } \\
(\%)\end{array}$ & Modelo & $\mathrm{C}_{0}$ & $C_{0}+C$ & $A(m)$ & GDE \\
\hline & \multicolumn{9}{|c|}{ Horizonte A } \\
\hline Areia $\left(\mathrm{g} \mathrm{kg}^{-1}\right)^{*}$ & 825 & 749 & 903 & 5 & Esf. & 163,4 & 885,400 & 135 & 18 \\
\hline Argila $\left(\mathrm{g} \mathrm{kg}^{-1}\right)$ & 94 & 29 & 148 & 31 & Gaus. & 375,0 & 906,900 & 164 & 41 \\
\hline $\mathrm{pH}\left(\mathrm{H}_{2} \mathrm{O}\right)$ & 7,0 & 5,9 & 8,2 & 8 & Esf. & 0,010 & 0,259 & 144 & 4 \\
\hline$C E\left(d S m^{-1}\right)$ & 0,1 & 0,0 & 0,2 & 37 & Esf. & 0,000 & 0,001 & 148 & 28 \\
\hline $\mathrm{Na}\left(\mathrm{cmol}_{\mathrm{c}} \mathrm{dm}^{-3}\right)$ & 0,0 & 0,0 & 0,1 & 51 & EPP & 0,000 & 0,000 & 317 & 100 \\
\hline $\mathrm{K}\left(\mathrm{cmol}_{\mathrm{c}} \mathrm{dm}^{3}\right)$ & 0,1 & 0,0 & 0,4 & 70 & Esf. & 0,002 & 0,010 & 147 & 17 \\
\hline $\mathrm{H}+\mathrm{Al}\left(\mathrm{cmol}_{\mathrm{c}} \mathrm{dm}^{-3}\right)$ & 1,4 & 0,3 & 3,1 & 38 & Esf. & 0,013 & 0,262 & 144 & 5 \\
\hline $\mathrm{Al}\left(\mathrm{cmol}_{\mathrm{c}} \mathrm{dm}^{-3}\right)$ & 0,1 & 0,0 & 0,2 & 63 & Gau. & 0,002 & 0,003 & 554 & 51 \\
\hline $\mathrm{Ca}\left(\mathrm{cmol}_{\mathrm{c}} \mathrm{dm}^{-3}\right)$ & 1,7 & 0,1 & 3,5 & 49 & Esf. & 0,052 & 0,986 & 464 & 5 \\
\hline $\mathrm{Mg}\left(\mathrm{cmol}_{\mathrm{c}} \mathrm{dm}^{-3}\right)$ & 0,8 & 0,4 & 1,9 & 45 & Gau. & 0,038 & 0,153 & 220 & 25 \\
\hline $\mathrm{T}\left(\mathrm{cmol}_{\mathrm{c}} \mathrm{dm}^{-3}\right)$ & 4,1 & 2,4 & 7,0 & 23 & Esf. & 0,322 & 1,050 & 455 & 31 \\
\hline \multirow[t]{2}{*}{$\vee(\%)^{*}$} & 64 & 32 & 89 & 24 & Gau. & 42,2 & 89,100 & 108 & 47 \\
\hline & \multicolumn{9}{|c|}{ Horizonte B } \\
\hline Areia $\left(\mathrm{g} \mathrm{kg}^{-1}\right)$ & 713 & 629 & 844 & 7 & Gau. & 1602,1 & 3226,2 & 400 & 50 \\
\hline Argila $\left(\mathrm{g} \mathrm{kg}^{-1}\right)$ & 179 & 52 & 259 & 31 & Exp. & 625,8 & 3536,1 & 420 & 18 \\
\hline $\mathrm{pH}\left(\mathrm{H}_{2} \mathrm{O}\right)$ & 6,3 & 4,6 & 7,9 & 14 & Esf. & 0,031 & 0,862 & 194 & 4 \\
\hline $\mathrm{CE}\left(\mathrm{dS} \mathrm{m}^{-1}\right)$ & 0,1 & 0,0 & 0,4 & 77 & Exp. & 0,000 & 0,002 & 275 & 8 \\
\hline $\mathrm{Na}\left(\mathrm{cmol}_{\mathrm{c}} \mathrm{dm}^{-3}\right)$ & 0,0 & 0,0 & 0,1 & 71 & Esf. & 0,000 & 0,001 & 262 & 35 \\
\hline $\mathrm{K}\left(\mathrm{cmol}_{\mathrm{c}} \mathrm{dm}^{-3}\right)$ & 0,1 & 0,0 & 0,4 & 80 & Esf. & 0,001 & 0,005 & 169 & 26 \\
\hline $\mathrm{H}+\mathrm{Al}\left(\mathrm{cmol}_{\mathrm{c}} \mathrm{dm}^{-3}\right)$ & 1,3 & 0,3 & 4,5 & 56 & Exp. & 0,076 & 0,579 & 221 & 13 \\
\hline $\mathrm{Al}\left(\mathrm{cmol}_{\mathrm{c}} \mathrm{dm}^{-3}\right)$ & 0,1 & 0,0 & 0,6 & 76 & Esf. & 0,004 & 0,009 & 434 & 45 \\
\hline $\mathrm{Ca}\left(\mathrm{cmol}_{\mathrm{c}} \mathrm{dm}^{-3}\right)$ & 0,8 & 0,1 & 1,9 & 71 & Esf. & 0,018 & 0,466 & 434 & 4 \\
\hline $\mathrm{Mg}\left(\mathrm{cmol}_{\mathrm{c}} \mathrm{dm}^{-3}\right)$ & 1,1 & 0,7 & 1,5 & 15 & Exp. & 0,007 & 0,036 & 444 & 19 \\
\hline $\mathrm{T}\left(\mathrm{cmol}_{\mathrm{c}} \mathrm{dm}^{-3}\right)$ & 3,4 & 2,1 & 6,1 & 24 & Gau. & 0,416 & 0,960 & 497 & 43 \\
\hline$\vee(\%)$ & 63 & 27 & 91 & 22 & Gau. & 53,20 & 189,7 & 137 & 28 \\
\hline
\end{tabular}

Para o quarto nível categórico, a textura do solo foi o atributo determinante na classificação. Portanto foram utilizados os mapas de areia e de argila que levaram em consideração a média dos dados dos dois horizontes e permitiram a definição do $4^{\circ}$ nível categórico (subgrupos) dos solos, classificandoos Distrocoesos em arênicos (> $700 \mathrm{~g} \mathrm{~kg}^{-1}$ de areia e $<150 \mathrm{~g} \mathrm{~kg}^{-1}$ de argila) ou típicos, e os Eutrocoesos em típicos.

Para a formação do mapa pedológico até o quarto nível categórico foi utilizado álgebra de mapas conforme realizado por Silva et al. (2016) com os critérios anteriormente discutidos utilizando-se a ferramenta "Raster Calculater" e a ferramenta de busca "Con" (ou se) obtendo a seguinte condicional: Con(("V\%">50\%), "PAex", Con(("V\%"<50\%) \& ("areia">700 $\mathrm{g} \mathrm{kg}^{-1}$ ) \& ("argila"> $\left.150 \mathrm{~g} \mathrm{~kg}^{-1}\right)$, "PAdxl", Con(("V\%"<50\%) \& ("areia"<700 $\mathrm{g} \mathrm{kg}^{-1}$ ) \& ("argila">150 $\mathrm{g} \mathrm{kg}^{-1}$ ), "PAdx1", Con(("V\%"<50\%) \& ("areia"<700 g kg-1) \& ("argila"<150 $\mathrm{g} \mathrm{kg}^{-1}$ ), "PAdx1","PAdx2",))))".

O Argissolo Amarelo Eutrocoeso típico compreende a maior parte da área, abrangendo uma porcentagem de $92,57 \%$, o Argissolo Amarelo Distrocoeso típico encontra-se em 5,93 \% da área e o arênico em 1,49 \% (Figura 1).

O presente trabalho confirma que - uso de métodos geoestatísticos torna o 
processo de mapeamento de solos em nível ultradetalhado mais eficiente concordando com os resultados encontrados por Kempen et al. (2012) em mapeamento de solos na Holanda. Isso ocorre porque o uso de técnicas geoestatísticas e demais técnicas quantitativas torna o mapeamento mais rápido devido a informatização e menos subjetivo quanto a definição das unidades de mapeamento
(Chagas et al., 2010). Adicionalmente, sabendose que a relação entre produtividade das culturas e os atributos do solo é um processo dinâmico no espaço e no tempo, técnicas geoestatísticas são fundamentais para entender as relações de causa/efeito entre atributos do solo e produtividade das culturas (Rodrigues et al., 2012) e com isso possibilitar a extrapolação de resultados de pesquisa.

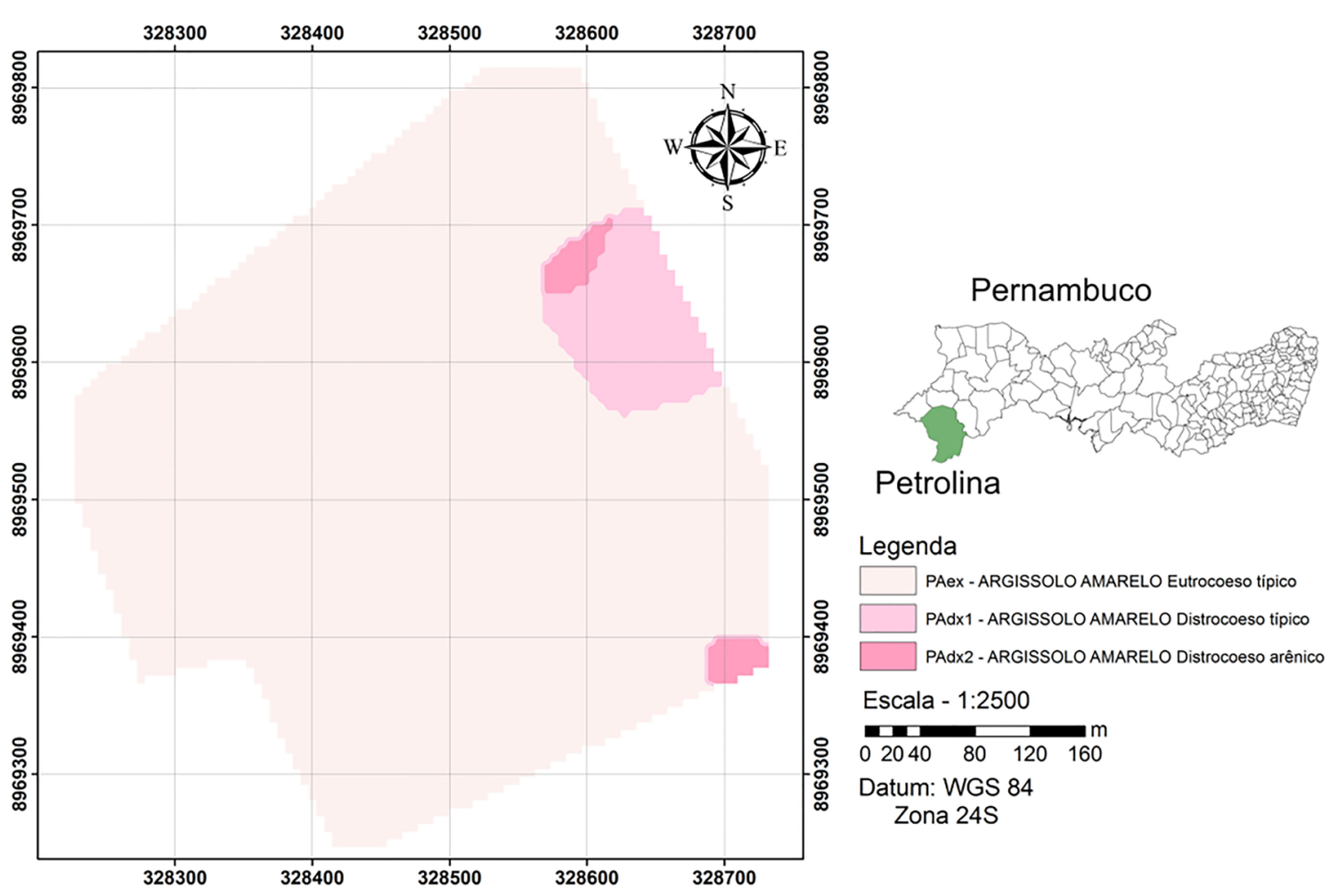

Figura 1. Mapa das Classes de Solos em uma área de experimentação agrícola em Petrolina-PE.

\section{Referências}

Chagas, C.D.S., Fernandes Filho, E.I., Vieira, C.A.O., Schaefer, C.E.G.R., Carvalho Júnior, W.D. 2010. Atributos topográficos e dados do Landsat7 no mapeamento digital de solos com uso de redes neurais. Pesquisa Agropecuária Brasileira 45: 497-507.

Dalmolin, R.S.D., Ten Caten, A. 2015. Mapeamento Digital: nova abordagem em levantamento de solos. Investigación Agraria 17 (2): 77-86.

Donagema, G.K., Campos, D.V.B., Calderano, S.B., Teixeira, W.G., Viana, J.H.M. 2011. Manual de Métodos de Análise de Solo. 2. ed. Embrapa Solos, Rio de Janeiro, Brasil. 230 p.

IBGE. 2015. Manual técnico de pedologia. 3. ed. IBGE, Rio de Janeiro, Brasil. 430 p.

Kempen, B., Brus, D.J., Stoorvogel, J.J., Heuvelink, G.B.M., De Vries, F. 2012. Efficiency Comparison of Conventional and Digital Soil Mapping for
Updating Soil Maps. Soil Science Society of America Journal 76: 2097-2115.

Oliver, M.A., Webster, R. 2014. A tutorial guide to geoestatistics: computing and modelling variograms and kriging. Catena 113: 56-59.

Rodrigues, M.S., Corá, J.E., Fernandes, C. 2012. Spatial relationships between soil attributes and corn yield in no-tillage system. Revista Brasileira de Ciência do Solo 36: 599-609.

Santos, R.D., Lemos, R.C., Santos, H.G., Ker, J.C., Anjos, L.H.C., Shimizu, S.H. 2013a. Manual de descrição e coleta de solo no campo. 6. ed. Sociedade Brasileira de Ciência do Solo, Viçosa, Brasil. 100 p.

Santos, H.G., Jacomine, P.K.T., Anjos L.H.C., Oliveira, V.A., Oliveira, J.B., Coelho, M.R., Lumbreras, J.F., Cunha, T.J.F. 2013b. Sistema brasileiro de classificação de solos. $3^{a}$ ed. Embrapa Solos, Rio de Janeiro, Brasil. 353 p. 
Shi, X.Z., Yu, D.S., Yang, G.X., Wang, H.J., Sun, W.X., Du, G.H., Gong, Z.T. 2006. Cross-Reference Benchmarks for Translating the Genetic Soil Classification of China into the Chinese Soil Taxonomy. Pedosphere 16: 147-153.

Silva, S.H.G., Menezes, M.D.D., Owens, P.R., Curi, N. 2016. Retrieving pedologist's mental model from existing soil map and comparing data mining tools for refining a larger area map under similar environmental conditions in Southeastern Brazil. Geoderma 267: 65-77.

Silva, C.C.D., Coelho, R.M., Oliveira, S.R.D.M., Adami, S.F. 2013. Mapeamento pedológico digital da folha Botucatu (SF-22-Z-B-VI-3): treinamento de dados em mapa tradicional e validação de campo. Revista Brasileira de Ciência do Solo 37: 846-857.

Ten Caten, A., Dalmolin, R.S.D., MendonçaSantos, M.D.L., Giasson, E. 2012. Mapeamento digital de classes de solos: características da abordagem brasileira. Ciência Rural 42: 19891997.

Vieira, S.R., Carvalho, J.R.P., Ceddia, M.B., González, A.P. 2010. Detrending non stationary data for geostatistical applications. Bragantia 69: 1-8. 